\title{
Recurrence Relations for the Indefinite Integrals of the Associated Legendre Functions
}

\author{
By A. R. DiDonato
}

Abstract. Two recurrence relations are derived for the computation of the integral of the associated Legendre functions of real argument and integer order and degree.

The objective of this note is to develop recurrence relations for the integral

$$
S_{n}^{m}(x) \equiv \int_{a}^{x} P_{n}^{m}(t) d t, \quad|x| \leqslant 1,-1 \leqslant a<1,
$$

on the indices $n \geqslant m \geqslant 0$, where $P_{n}^{m}(x)$ denotes the classical associated Legendre function of degree $n$ and order $m$ with real argument $x$. We use the definition

$$
P_{n}^{m}(x) \equiv\left(1-x^{2}\right)^{m / 2} \frac{d^{m}}{d x^{m}}\left[P_{n}(x)\right], \quad[4, \text { p. 323] }
$$

with $P_{n}^{0}(x) \equiv P_{n}(x)$, the Legendre polynomial of degree $n$, i.e.,

$$
P_{n}^{\prime}(x) \equiv \frac{1}{2^{n} n !} \frac{d^{n}}{d x^{n}}\left(x^{2}-1\right)^{n}, \quad[4, \text { p. 303] }
$$

The indices $n$ and $m$ are treated as nonnegative integers throughout, and $a$ is a constant usually taken as zero or minus one.

The final expressions are given below by (12) and (17) and in a particular normalized form in $(\overline{12})$ and $(\overline{17})$. It is unlikely these relations are new, however we have not been able to find them in the literature, and their absence is conspicuous in such classical texts as [1], [4].* The need for such relations occurs in geodesy studies, [3], [5].

We first list for easy reference some well-known relations, $[1$, p. 1325]:

$$
\begin{gathered}
P_{n+1}=\frac{2 n+1}{n+1} x P_{n}-\frac{n}{n+1} P_{n-1}, \quad n \geqslant 0,\left(P_{-1} \equiv 0\right), \\
P_{n+1}^{m}=\frac{2 n+1}{n-m+1} x P_{n}^{m}-\frac{n+m}{n-m+1} P_{n-1}^{m}, \quad n \geqslant m \geqslant 0, \\
P_{n}^{n}=\frac{(2 n) !}{2^{n} n !}\left(1-x^{2}\right)^{n / 2}, \quad P_{n+1}^{n}=(2 n+1) x P_{n}^{n}, \\
\left(1-x^{2}\right) \frac{d}{d x}\left(P_{n}^{m}\right)=(n+1) x P_{n}^{m}-(n-m+1) P_{n+1}^{m},
\end{gathered}
$$

Received February 5, 1979; revised August 7, 1981.

1980 Mathematics Subject Classification. Primary 33A45.

*After this work was completed, it was brought to our attention that (12) had recently appeared in the literature, [2]. It was decided to include our own derivation since it is different and more concise than the one in [2]. 


$$
\begin{aligned}
P_{n+1}^{m+1}= & P_{n-1}^{m+1}+(n+m)(n+m-1) P_{n-1}^{m-1} \\
& -(n-m+1)(n-m+2) P_{n+1}^{m-1}
\end{aligned}
$$

$$
\sqrt{1-x^{2}} P_{n}^{m}
$$

$$
=\frac{1}{2 n+1}\left[(n+m)(n+m-1) P_{n-1}^{m-1}-(n-m+1)(n-m+2) P_{n+1}^{m-1}\right],
$$

$$
\frac{d}{d x}\left[\left(1-x^{2}\right)^{m / 2} P_{n}^{m}\right]=-(n-m+1)(n+m)\left(1-x^{2}\right)^{(m-1) / 2} P_{n}^{m-1}
$$

The desired recurrence relation on $n$ is obtained without much difficulty. By first integrating (7) and then carrying out an integration by parts on the left we get

1) $\left.\left(1-t^{2}\right) P_{n}^{m}(t)\right|_{a} ^{x}+2 \int_{a}^{x} t P_{n}^{m}(t) d t=(n+1) \int_{a}^{x} t P_{n}^{m}(t) d t-(n-m+1) S_{n+1}^{m}$.

Then integrating (5) and using it in (11) gives the result we want, namely,

$$
\begin{array}{r}
S_{n+1}^{m}=\frac{(n-1)(n+m)}{(n+2)(n-m+1)} S_{n-1}^{m}-\left.\frac{2 n+1}{(n+2)(n-m+1)}\left(1-t^{2}\right) P_{n}^{m}(t)\right|_{a} ^{x}, \\
0 \leqslant m \leqslant n .
\end{array}
$$

The steps leading to a recurrence relation on $m$ for (1) begin with the integration of (8). The result is

$$
\begin{aligned}
S_{n+1}^{m+1}= & S_{n-1}^{m+1}+(n+m)(n+m-1) S_{n-1}^{m-1} \\
& -(n-m+1)(n-m+2) S_{n+1}^{m-1}
\end{aligned}
$$

Now replacing $m$ by $(m-1)$ in (12) and using some obvious modifications gives

$$
\begin{aligned}
(n+ & m-1)(n+m) S_{n-1}^{m-1} \\
& =\frac{(2 n+1)(n+m)}{n-1}\left[\frac{(n-m+2)(n+2)}{2 n+1} S_{n+1}^{m-1}+\left.\left(1-t^{2}\right) P_{n}^{m-1}(t)\right|_{a} ^{x}\right] .
\end{aligned}
$$

Inserting this result in (13), we have

$$
\begin{aligned}
S_{n+1}^{m+1}= & S_{n-1}^{m+1}+\frac{(2 n+1)(m+1)(n-m+2)}{n-1} S_{n+1}^{m-1} \\
& +\left.\frac{(2 n+1)(n+m)}{n-1}\left(1-t^{2}\right) P_{n}^{m-1}(t)\right|_{a} ^{x}
\end{aligned}
$$

In a similar way, we replace $m$ by $(m+1)$ in (12) to get

$$
\frac{(n-1)(n+m+1)}{2 n+1} S_{n-1}^{m+1}=\frac{(n-m)(n+2)}{2 n+1} S_{n+1}^{m+1}+\left.\left(1-t^{2}\right) P_{n}^{m+1}(t)\right|_{a} ^{x} .
$$

Then using this result in (15) yields

$$
\begin{aligned}
S_{n+1}^{m+1}=\frac{1}{m-1} & \left\{(m+1)(n-m+2)(n+m+1) S_{n+1}^{m-1}\right. \\
& \left.+\left.\left(1-t^{2}\right)\left[P_{n}^{m+1}(t)+(n+m)(n+m+1) P_{n}^{m-1}(t)\right]\right|_{a} ^{x}\right\} .
\end{aligned}
$$


Replacing $n+1$ by $n$ in (16) gives the desired result, namely

$$
\begin{aligned}
S_{n}^{m+1}=\frac{1}{m-1}\{ & (m+1)(n-m+1)(n+m) S_{n}^{m-1} \\
& \left.+\left.\left(1-t^{2}\right)\left[P_{n-1}^{m+1}(t)+(n+m-1)(n+m) P_{n-1}^{m-1}(t)\right]\right|_{a} ^{x}\right\}, \\
& \quad 2 \leqslant m \leqslant n .
\end{aligned}
$$

To start the recurrence relations (12) and (17) we can use

$$
\begin{gathered}
P_{0}^{0}=1, \quad P_{1}^{0}=x, \quad P_{2}^{0}=\frac{1}{2}\left(3 x^{2}-1\right), \quad P_{1}^{1}=\sqrt{1-x^{2}} \\
P_{n+1}=\frac{2 n+1}{n+1} x P_{n}-\frac{n}{n+1} P_{n-1}, \quad n \geqslant 0, P_{-1} \equiv 0,(\text { from }(4)), \\
S_{0}^{0}=x-a, \quad S_{1}^{1}=\left.\frac{1}{2}\left[t \sqrt{1-t^{2}}+\sin ^{-1} t\right]\right|_{a} ^{x} \\
S_{1}^{0}=\frac{x^{2}-a^{2}}{2},
\end{gathered}
$$

and, in general,

$$
\begin{gathered}
S_{n}^{0}=\left.\frac{1}{2 n+1}\left[P_{n+1}(t)-P_{n-1}(t)\right]\right|_{a} ^{x},{ }^{* *} \\
S_{n}^{1}=\frac{(n-2) n}{(n-1)(n+1)} S_{n-2}^{1}-\left.\frac{2 n-1}{(n-1)(n+1)}\left(1-t^{2}\right) P_{n-1}^{1}(t)\right|_{a} ^{x}, \\
n \geqslant 2,(\text { from }(12)),
\end{gathered}
$$

$$
S_{n}^{2}=-2 S_{n}^{0}+\left.\left[(n+3) t P_{n}(t)-(n+1) P_{n+1}(t)\right]\right|_{a} ^{x} \quad(\text { from }(2),(9),(4)) \text {, }
$$

$$
\left\{\begin{array}{l}
P_{n+1}^{m}=\frac{2 n+1}{n-m+1} x P_{n}^{m}-\frac{n+m}{n-m+1} P_{n-1}^{m}, \quad n \geqslant 0, n \geqslant m, \\
P_{n+1}^{n+1}=(2 n+1) \sqrt{1-x^{2}} p_{n}^{n} \quad(\text { from (9) and (8)) }
\end{array}\right.
$$

(26) $S_{n}^{n}=\frac{1}{n+1}\left[n(2 n-3)(2 n-1) S_{n-2}^{n-2}+\left.t P_{n}^{n}(t)\right|_{a} ^{x}\right]$

$$
S_{n+1}^{n}=-\left.\frac{1}{n+2} \sqrt{1-t^{2}} P_{n+1}^{n+1}(t)\right|_{a} ^{x}
$$

Normalizations of $P_{n}^{m}(x)$ and $S_{n}^{m}(x)$ may be needed in order to keep the results of the recurrence relations from becoming excessively large. Since

$$
\int_{-1}^{1}\left[P_{n}^{m}(t)\right]^{2} d t=\left(\frac{2}{2 n+1}\right) \frac{(n+m) !}{(n-m) !},
$$

we define normalized Legendre functions $\bar{P}_{n}^{m}(x)$ by

$$
\bar{P}_{n}^{m} \equiv\left[\frac{2 n+1}{2} \frac{(n-m) !}{(n+m) !}\right]^{1 / 2} P_{n}^{m}(x) .
$$

\footnotetext{
**'The result in (22) follows from integrating (10), with $m=1$, and then using (9).
} 
Accordingly, for (5), (12), and (17), respectively, we have their normalized forms, where we number a normalized equation the same as the corresponding unnormalized equation except for the addition of an overbar.

$$
\bar{P}_{n+1}^{m}(x)=\left[\frac{(2 n+3)(2 n+1)}{(n+m+1)(n-m+1)}\right]^{1 / 2} x \bar{P}_{n}^{m}(x)
$$

$-\left[\frac{(2 n+3)(n+m)(n-m)}{(2 n-1)(n+m+1)(n-m+1)}\right]^{1 / 2} \bar{P}_{n-1}^{m}(x)$,

$$
0 \leqslant m<n+1
$$

$$
\bar{S}_{n+1}^{m}(x)=\frac{n-1}{n+2}\left[\frac{(2 n+3)(n+m)(n-m)}{(2 n-1)(n+m+1)(n-m+1)}\right]^{1 / 2} \bar{S}_{n-1}^{m}(x)
$$

$$
-\left.\frac{\left(1-t^{2}\right)}{n+2}\left[\frac{(2 n+3)(2 n+1)}{(n+m+1)(n-m+1)}\right]^{1 / 2} \bar{P}_{n}^{m}(t)\right|_{a} ^{x},
$$

$\bar{S}_{n}^{m+1}(x)=\frac{1}{m-1}\left\{(m+1)\left[\frac{(n+m)(n-m+1)}{(n+m+1)(n-m)}\right]^{1 / 2} \bar{S}_{n}^{m-1}\right.$

$$
\begin{aligned}
& +\left.\left(1-t^{2}\right)\left[\frac{(2 n+1)(n-m-1)}{(2 n-1)(n+m+1)}\right]^{1 / 2} \bar{P}_{n-1}^{m+1}(t)\right|_{a} ^{x} \\
& \left.+\left.\left(1-t^{2}\right)\left[\frac{(2 n+1)(n+m)(n+m-1)}{(2 n-1)(n+m+1)(n-m)}\right]^{1 / 2} \bar{P}_{n-1}^{m-1}(t)\right|_{a} ^{x}\right\} \\
& \quad 2 \leqslant m<n,
\end{aligned}
$$

where

$$
\bar{S}_{n}^{m}(x) \equiv\left[\frac{2 n+1}{2} \frac{(n-m) !}{(n+m) !}\right]^{1 / 2} S_{n}^{m}(x)
$$

The starting relations (18)-(27) in normalized form are given in the same order.

$$
\begin{aligned}
\bar{P}_{0}=1 / \sqrt{2}, \quad \bar{P}_{1}(x) & =(3 / 2)^{1 / 2} x, \quad \bar{P}_{2}(x)=(5 / 2)^{1 / 2} \frac{1}{2}\left(3 x^{2}-1\right) \\
\bar{P}_{1}^{1}(x)=\left[3\left(1-x^{2}\right)\right]^{1 / 2} / 2, & \\
\bar{P}_{n+1}(x)= & {[(2 n+3)(2 n+1)]^{1 / 2} } \\
n+1 & \bar{P}_{n}(x) \\
& -\frac{n}{n+1}\left(\frac{2 n+3}{2 n-1}\right)^{1 / 2} \bar{P}_{n-1}(x), \quad n \geqslant 0,
\end{aligned}
$$

$$
\bar{S}_{0}^{0}=\frac{1}{\sqrt{2}}(x-a), \quad \bar{S}_{1}^{1}=\left.\frac{\sqrt{3}}{4}\left[t \sqrt{1-t^{2}}+\sin ^{-1} t\right]\right|_{a} ^{x}
$$

$$
\bar{S}_{1}^{0}=\frac{(3 / 2)^{1 / 2}}{2}\left(x^{2}-a^{2}\right) \text {, }
$$




$$
\begin{gathered}
\bar{S}_{n}^{0}=\left.\left\{\left[\frac{1}{(2 n+1)(2 n+3)}\right]^{1 / 2} \bar{P}_{n+1}(t)-\left[\frac{1}{(2 n+1)(2 n-1)}\right]^{1 / 2} \bar{P}_{n-1}(t)\right\}\right|_{a} ^{x}, \\
\bar{S}_{n}^{1}=\frac{n-2}{n+1}\left[\frac{(2 n+1) n(n-2)}{(2 n-3)(n+1)(n-1)}\right]^{1 / 2} \bar{S}_{n-2}^{1} \\
-\left.\frac{\left(1-t^{2}\right)}{n+1}\left[\frac{(2 n+1)(2 n-1)}{(n+1)(n-1)}\right]^{1 / 2} \bar{P}_{n-1}^{1}(t)\right|_{a} ^{x}
\end{gathered}
$$

$$
\bar{S}_{n}^{2}=\frac{1}{[(n+2)(n+1) n(n-1)]^{1 / 2}}
$$

$$
\times\left\{-2 \bar{S}_{n}^{0}+\left.\left[(n+3) t \bar{P}_{n}(t)-(n+1)\left(\frac{2 n+1}{2 n+3}\right)^{1 / 2} \bar{P}_{n+1}(t)\right]\right|_{a} ^{x}\right\},
$$

$$
\begin{gathered}
\bar{P}_{n+1}^{n+1}(x)=\left[\frac{2 n+3}{2 n+2}\right]^{1 / 2}\left(1-x^{2}\right)^{1 / 2} \bar{P}_{n}^{n}(x), \\
\bar{S}_{n}^{n}=\frac{1}{n+1}\left\{\left[\frac{n(2 n-1)(2 n+1)}{4(n-1)}\right]^{1 / 2} \bar{S}_{n-2}^{n-2}+\left.t \bar{P}_{n}^{n}\right|_{a} ^{x}\right\}, \\
\bar{S}_{n+1}^{n}=-\left.\frac{1}{n+2}[2(n+1)]^{1 / 2}\left(1-t^{2}\right)^{1 / 2} \bar{P}_{n+1}^{n+1}(t)\right|_{a} ^{x} .
\end{gathered}
$$

Acknowledgement. The author is indebted to B. Zondek for proposing the problem, and to W. M. Robertson for calling [2] to his attention.

Naval Surface Weapons Center

Dahlgren Laboratory

Dahlgren, Virginia 22448

1. P. M. Morse \& H. Feshbach, Methods of Theoretical Physics, Vol. II, McGraw-Hill, New York, 1953.

2. M. K. PAUL, "Recurrence relations for integrals of associated Legendre functions," Bull. Géodésique, v. 52, 1978, pp. $177-190$.

3. W. M. Robertson, Spherical Geodetic Transformations, Vol. I of II, Report No. R-1181, The Charles Stark Draper Laboratories, Inc., Cambridge, Mass. 02139, September 1978.

4. E. T. Whittaker \& G. N. Watson, A Course of Modern Analysis, Cambridge Univ. Press, Oxford, 1952.

5. B. ZondeK, Aggregation Errors of Cell-Averaged Geoid Heights, Naval Surface Weapons Center, Dahlgren Laboratory, TR-3608, April 1977. 